University at Albany, State University of New York

Scholars Archive

\title{
Prevalence of Adverse Childhood Experiences (ACEs) Among Child Service Providers
}

\author{
Nina Esaki \\ Sanctuary Institute \\ Heather Larkin Holloway \\ University at Albany, State University of New York, hlarkin@albany.edu
}

Follow this and additional works at: https://scholarsarchive.library.albany.edu/ssw_sw_scholar

Part of the Social Work Commons

\section{Recommended Citation}

Esaki, Nina and Larkin Holloway, Heather, "Prevalence of Adverse Childhood Experiences (ACEs) Among Child Service Providers" (2013). Social Welfare Faculty Scholarship. 2.

https://scholarsarchive.library.albany.edu/ssw_sw_scholar/2

This Article is brought to you for free and open access by the Social Welfare at Scholars Archive. It has been accepted for inclusion in Social Welfare Faculty Scholarship by an authorized administrator of Scholars Archive. For more information, please contact scholarsarchive@albany.edu. 


\title{
Prevalence of Adverse Childhood Experiences (ACEs) Among Child Service Providers
}

\author{
Nina Esaki \& Heather Larkin
}

\begin{abstract}
Despite increasing evidence in the public health field about the prevalence of adverse childhood experiences (ACEs) in the general population, little is known about the prevalence of ACEs among social service providers. Trauma backgrounds may influence both worker susceptibility to vicarious traumatization as well as clinical decision making. Similarly, individuals with trauma backgrounds are vulnerable to re-enactment of their history. With pressure on social service agencies to demonstrate successful client outcomes, it is critical that agencies explore factors that influence high-quality care. This exploratory study is the first investigation of ACE prevalence among workers in an agency that provides residential treatment, day treatment, and schooling for children with histories of trauma. Study results suggest a high prevalence of ACEs among these workers.
\end{abstract}

\section{IMPLICATIONS FOR PRACTICE}

- There is a need to further explore the prevalence of ACEs among human service agency workers since this issue, if left unaddressed, may affect provision of high quality services.

- Agencies may benefit by the creation of restorative organizational cultures to improve overall functioning of the agency and subsequent quality of services.

$\Lambda$ dverse childhood experiences (ACEs), which include physical and sexual abuse, physical and emotional neglect, poverty, parental psychopathology, and conflict between parents, are common in the general population (Chartier, Walker, \& Naimark, 2010). Research from high-income countries estimates that $4-16 \%$ of children experience severe parental violence and that $10-20 \%$ of children witness interparental violence. Sexual abuse is experienced by $15-30 \%$ of girls and $5-15 \%$ of boys (Gilbert et al., 2009).

To examine whether a history of ACEs was common in a randomly selected population in the United States, the Centers for Disease Control and Prevention (CDC) analyzed information from 26,229 adults in five states using the 2009 ACE module of the Behavioral Risk Factor Surveillance System (BRFSS; Bynum et al., 2010). ACEs in the BRFSS included verbal, physical, or sexual abuse, as well as family dysfunction (e.g., an incarcerated, mentally ill, or substance-abusing family member; domestic violence; or absence of a parent because of divorce or separation). The results of the analysis indicated that, overall, $59.4 \%$ of respondents reported having at least one ACE, and $8.7 \%$ reported five or more ACEs. Despite increasing knowledge about the prevalence of trauma in the general population, little is known about the prevalence of ACEs among social service providers.

\section{ACEs Among Social Workers}

Knowledge about the prevalence of ACEs among social workers has been limited to studies conducted with social work students. In their seminal social work study, Black, Jeffreys, and Hartley (1993) found support for a link between psychosocial trauma in the early life of social work students and their choice of a social work career (Lyter, 2008). Social work students reported a significantly higher frequency of early life family trauma when compared with a sample of business students. Traumatic events occurring before the age of 21 included alcohol or drug abuse; physical, sexual, or emotional abuse; physical or mental illness; suicide; and death of a family member.

Likewise, Rompf and Royse (1994) found that psychosocial trauma in early life was associated with choice of social work as a career. The psychosocial trauma from which social work students suffered more than non-social work students included unhappily married parents, emotional problems, alcoholism, drug addiction, and child abuse or neglect within their families while growing up. Russel, Gill, Coyne, and Woody (1993) found that master of social work students, more frequently than other graduate students, came from families in which alcohol or drug abuse was a problem. In fact, $73 \%$ had experienced one or more problems that defined a dysfunctional family, a figure significantly greater than the comparable percentage for graduate students in education or business (Russel et al., 1993). Though the studies are limited in number, the findings suggest that social workers may have a higher ACE prevalence than the general population.

\section{Personal Histories of Trauma and Implications for Social Work}

Although limited, some studies have suggested that personal histories of sexual abuse may influence clinical perceptions and judgments, in that social workers who reported their own abuse or witnessed another's abuse 
were more likely to believe the sexual abuse allegations of their clients compared with social workers who reported no history of abuse (Cromer \& Freyd, 2009; Jackson \& Nuttall, 1994). Similarly, individuals with trauma backgrounds are vulnerable to re-enactment of their history. In behavioral re-enactment of the trauma, the self may play the role of either victim or victimizer (van der Kolk, 1989).

Likewise, it is increasingly recognized that helping professionals are likely to be traumatized as a result of their work with challenging clients, such as those with histories of trauma (Knight, 2010). Helping professionals can experience "indirect trauma" that may take on the forms of secondary traumatic stress (STS), vicarious trauma (VT), and compassion fatigue (Knight, 2010).

Secondary traumatic stress. STS relates to the behaviors and emotions resulting from knowing about a traumatizing event experienced by a client and the stress resulting from helping or wanting to help the individual (Figley, 1995). Results of several studies indicate that a personal trauma history, particularly in childhood, is a significant risk factor for STS (Ghahramanlou \& Brodbeck, 2000; Nelson-Gardell \& Harris, 2003). Building upon prior study findings (Follette, Polusny, \& Milbeck, 1994; Schauben \& Frazier, 1995), Ga-Young (2011) found that social workers who received more support from their coworkers, supervisors, and work teams demonstrated lower levels of STS.

Vicarious trauma. VT (McCann \& Pearlman, 1990) focuses on the cognitive schemas or core beliefs of the therapist and the way in which these may change as a result of empathic engagement with the client and exposure to the traumatic imagery presented by clients (Bober \& Regehr, 2005). This may cause a disruption in the therapist's view of self, others, and the world in general (McCann \& Pearlman, 1990; Sabin-Farrell \& Turpin, 2003). Personal trauma backgrounds may influence therapist susceptibility to VT (Pearlman \& Mac Ian, 1995). As reviewed by Trippany, White Kress, and Wilcoxen (2004), the impact of VT on counselors, if unacknowledged, can present ethical concerns (Saakvitne \& Pearlman, 1996). The potential for clinical error and therapeutic impasse increases as the vulnerability that counselors experience increases. The disruptions in cognitive schemas may lead to counselors compromising therapeutic boundaries (e.g., forgotten appointments, unreturned phone calls, inappropriate contact, abandonment, and sexual or emotional abuse of clients). Counselors may feel anger toward their clients when they have not complied with some idealized response to therapy (Herman, 1992). Counselors may begin doubting their skill and knowledge and potentially lose focus on clients' strengths and resources (Herman, 1992). In addition, counselors may avoid discussion of traumatic material or be intrusive when exploring traumatic memories by probing for specific details of the client's abuse or pushing to identify or confront perpetrators before the client is ready (Munroe, 1995).

Compassion fatigue. Also used interchangeably with STS and VT, this is best defined as a syndrome consisting of a combination of the symptoms of STS and professional burnout (Adams, Boscarino, \& Figley, 2006; Bride, Radey, \& Figley, 2007; Figley, 1995). Compassion fatigue has recently emerged in the literature as a more general term describing the overall experience of emotional and physical fatigue that social service professionals experience due to the chronic use of empathy when treating patients who are suffering in some way (Figley, 2002; Rothschild \& Rand, 2006). Much like professional burnout, the experience of compassion fatigue tends to occur cumulatively over time; whereas VT and STS have more immediate onset (Newell \& MacNeil, 2010).

At the organizational level, there have been several risk factors for STS, VT, and compassion fatigue (Newell \& MacNeil, 2010). These factors include organizational setting and bureaucratic constraints, inadequate supervision, lack of availability of client resources, and lack of support from professional colleagues (Catherall, 1995, 1999; Dunkley \& Whelan, 2006; Sabin-Farrell \& Turpin, 2003). Given the possible negative effects that STS, VT, and compassion fatigue can have on the quality of services delivered, it is important to consider organizational culture and the effect of agency culture on individual workers (Catherall, 1995).

In support of informing the social services field about the prevalence of ACEs among direct and indirect care staff, a residential voluntary child welfare agency in the Northeast, in collaboration with a researcher from a university school of social welfare, developed a survey for agency staff to obtain information about personal histories of ACEs. Using the survey, this particular exploratory study was guided by the following questions: (a) What is the prevalence of ACEs within this sample? (b) Were there differences in ACEs by gender, type of position held (direct or indirect care), race, or age of the respondent?

\section{Method}

This cross-sectional survey was developed through the collaborative effort of a residential voluntary child welfare agency located in the Northeast and a researcher from a university school of social welfare. The agency provides residential treatment, day treatment, and schooling for children with histories of trauma. The purpose of the survey was to collect information regarding ACEs of all agency staff, both direct and indirect care, to explore the prevalence of ACEs among providers of child services. The university institutional review board approved all study protocols. 


\section{Sample}

All employees of the agency were invited to take an online survey in April 2011. Staff surveyed included two categories to classify their work duties in relation to client care: direct and indirect service. Direct service represented clinicians, milieu therapists, child care workers, home visitors, and teachers. Indirect service positions included administrative, executive, research, training, clerical, finance, human resources, and development staff. A total of 94 out of 360 employees (26\%) participated in the study. Table 1 summarizes the characteristics of the sample. Of the respondents, $79.1 \%$ were female and $20.9 \%$ were male; $59.8 \%$ represented direct care and $40.2 \%$ indirect care staff, and the race/ethnicity profile was $65.9 \%$ White (not Hispanic) origin, 17.6\% Hispanic, 7.7\% Black (not Hispanic) origin, 5.5\% Asian or Pacific Islander, and 3.3\% of mixed racial heritage. The ages of participants ranged from a low of 22 to a high of 68 , with an average age of 39 .

\section{Measurement}

The survey began with demographic questions including gender, type of position held (direct or indirect care), age, and race/ethnicity. The ACE questionnaire, based on a previously validated survey instrument (Dube, Williamson, Thompson, Felitti, \& Anda, 2004), is an abbreviated version (CDC, 2011) and was used in a recent study (Larkin \& Park, 2012). Details of the items included in the survey are presented in Table 2. Questions used to define emotional and physical abuse, as well as growing up with domestic violence, were adapted from the Conflict Tactics Scale (Straus \& Gelles, 1990); to measure emotional and physical neglect, the Childhood Trauma Questionnaire (Bernstein et al., 1994) was used. Questions used to assess sexual abuse were adapted from Wyatt (1985). For the remaining questions, the researchers chose the simplest wording possible to obtain reasonable information (R. Anda, personal communication, March 24, 2009). The ACE Score was determined by adding the number of "yes" responses to each of the categories $(0-10)$.

In order to determine the internal consistency reliability for the 10 items composing the ACE survey, a coefficient alpha was computed for the measure. The a coefficient was .734, indicating adequate reliability (Nunnally, 1978).

\section{Data Collection}

Given that this study was exploratory, only one agency was recruited for participation. Known challenges with survey response rates at this agency led researchers to pursue a study design that would maximize participation. First, the ACE Study survey was an abbreviated version of the original ACE survey, one that was successfully used in a recent study (Larkin \& Park, 2012). All employees received an e-mail introducing the study from the chief executive officer of the agency. Shortly thereafter, all
TABLE 1. Demographics of Sample

\begin{tabular}{lcc}
\hline Characteristics & $n$ & $\%$ \\
\hline Gender $(n=91)$ & 72 & 79.1 \\
Female & 19 & 20.9 \\
Male & & \\
Type of position $(n=87)$ & 52 & 59.8 \\
Direct care & 35 & 40.2 \\
Indirect care & & \\
Race/ethnicity ( $n=91)$ & 60 & 65.9 \\
White (not Hispanic) & 16 & 17.6 \\
Hispanic & 7 & 7.7 \\
Black (not Hispanic) & 5 & 5.5 \\
Asian or Pacific Islander & 3 & 3.3 \\
Mixed racial heritage &
\end{tabular}

Note. $N=94$; due to missing data, category totals do not add up to 94 .

\section{TABLE 2. ACEs Study Survey Items}

Did a parent or other adult in the household often or very often...swear at you, insult you, put you down, or humiliate you? Or act in a way that made you afraid that you might be physically hurt?

Did a parent or other adult in the household often or very often...push, grab, slap, or throw something at you? Or ever hit you so hard that you had marks or were injured?

Did anyone older than you ever...touch or fondle you or have you touch their body in a sexual way? Or attempt or actually have oral, anal, or vaginal intercourse with you?

Did you often or very often feel that...no one in your family loved you or thought you were important or special? Or your family didn't look out for each other, feel close to each other, or support each other?

Did you often or very often feel that...you didn't have enough to eat, had to wear dirty clothes, and had no one to protect you? Or your parents were too drunk or high to take care of you or take you to the doctor if you needed it?

Was a biological parent ever lost to you through divorce, abandonment, or other reason?

Was any parent, stepparent, or parent figure: Often or very often pushed, grabbed, slapped, or had something thrown at him or her? Or sometimes, often, or very often kicked, bitten, hit with a fist, or hit with something hard? Or ever repeatedly hit over at least a few minutes or threatened with a gun or knife?

Did you live with anyone who was a problem drinker or alcoholic or who used street drugs?

Was a household member depressed or mentally ill, or did a household member attempt suicide?

Did a household member go to prison?

Adapted from "Adverse Childhood Experiences (ACE), Service Use, and Service Helpfulness Among People Experiencing Homelessness" by H. Larkin and J. Park, 2012, Families in Society, 93(2), pp. 85-93. Copyright 2012 by Alliance for Children and Families. 
employees received an e-mail message with the informed consent form and link to the online SurveyMonkey ${ }^{\circ}$ survey. A reminder e-mail message was sent out a week later to encourage participation. Employees were first invited to participate in the survey in April 2011 and were given 3 weeks to complete the survey.

\section{Data Analysis}

Data were analyzed using SPSS 19.0. Descriptive analysis identified prevalence of each ACE category and total ACE Scores. All nonmissing values were used. Thus, if a respondent answered "yes" to any question, the response was included in the identification of the prevalence of the ACE as well as used to determine the total ACE Score. Correlational analysis was used to assess any significant association between age of respondent and ACE Score. Independent-samples $t$ tests provided results on any differences in ACE Scores by gender, type of position held (direct or indirect care), and race (White/ non-White).

\section{Results}

\section{Descriptive Analysis}

As presented in Table 3, close to three quarters of the respondents (70.1\%) reported $\geq 1$ ACE category; $53.8 \%$ reported $\geq 2$ ACE categories, and $15.9 \%$ reported $\geq 4$ ACEs. A more detailed breakdown of prevalence by ACE category is provided in Table 4 . The prevalence of each ACE category ranged from a high of $34.1 \%$ for having a household member who was depressed or mentally ill to a low of $5.9 \%$ for suffering from physical neglect. The mean ACE Score was 2.0.

Correlational analysis. Nonparametric Kendall's tau bivariate correlation analyses were conducted to determine any significant association between age and ACE

TABLE 3. ACEs Scores

\begin{tabular}{lcc}
\hline Number of ACEs & $n$ & $\%$ \\
\hline None & 26 & 29.9 \\
One & 14 & 16.1 \\
Two & 23 & 26.4 \\
Three & 10 & 11.5 \\
Four & 3 & 3.5 \\
Five & 2 & 2.3 \\
Six & 5 & 5.7 \\
Seven & 2 & 2.3 \\
Eight & 1 & 1.2 \\
Nine & 1 & 1.1 \\
Ten & 0 & 0.0 \\
\hline
\end{tabular}

Note. $N=94$; due to missing data, category totals do not add up to 94 .
Score. The results of the correlation analysis between age and ACE Score, $r=-.001, p=.991$, indicated no significant relationship.

\section{Independent-Samples t Tests}

Independent-samples $t$ tests were run to investigate any differences in ACE Score by type of position held, gender, or race (White/non-White). The independentsamples $t$ tests assuming equal variances and not assuming equal variances on all group measures were not significant. Using the suggestion for always reporting the $t$ value for unequal variances and, thereby avoiding the homogeneity-of-variance assumption (Green \& Salkind, 2005 , p. 171), the test by type of position was not significant, $t(84)=.32, p=.75$, as was the case with gender, $t(48.49)=-1.34, p=.19$, and race, $t(84)=1.07, p=.29$. Thus, ACE Scores for this sample did not differ by any of the respondent demographic characteristics.

\section{Discussion}

The study results suggest a high prevalence of ACEs among workers in an agency serving children with histories of trauma. Approximately $70 \%$ of participating workers in this child-serving agency reported at least one of the ACE categories, $54 \%$ reported two or more ACE categories, and nearly $16 \%$ reported four or more ACE categories. Thus, ACEs were somewhat more prevalent among workers in this sample than the largely middle-class participants in Wave II of the ACE Study (Dong et al., 2004; Dube et al., 2001). The second wave of the ACE Study, including all 10 items, found that $64 \%$ of respondents had ACE Scores of 1 or more, a prevalence rate considered high to those researchers (Felitti \& Anda, 2010). Whereas the Wave II ACE Study reported $38 \%$ of respondents with ACE Scores of 2 or more, the current study has a notably

TABLE 4. Prevalence of Individual Categories of ACEs $(N=94)$

\begin{tabular}{lcc}
\hline ACE categories & $n$ & $\%$ \\
\hline $\begin{array}{l}\text { Depressed/mentally ill household } \\
\text { member }\end{array}$ & 29 & 34.1 \\
$\begin{array}{l}\text { Humiliation/physical threat by } \\
\text { household member }\end{array}$ & 27 & 31.8 \\
$\quad$ Substance abusing household member & 23 & 27.4 \\
Parental loss through divorce or & 20 & 23.5 \\
$\quad$ bandonment & & \\
Sexual abuse & 20 & 23.5 \\
Physical abuse & 19 & 22.6 \\
Lack of support & 15 & 18.1 \\
Domestic violence & 9 & 10.7 \\
Incarceration & 6 & 7.1 \\
Neglect & 5 & 5.9 \\
\hline
\end{tabular}


higher percentage, 53\%, with ACE Scores of 2 or more. In the Wave II ACE Study, 15\% of participants reported 4 or more ACEs (Dong et al., 2004; Dube et al., 2001).

The current study found that growing up with a family member experiencing depression or mental illness was the most frequently reported category, accounting for $34 \%$ of the ACE categories reported by child service providers. In contrast, Dong et al. (2004) found substance abuse in the family to be the most commonly reported ACE category.

The higher rates of ACEs among workers in a childserving agency than the general population may be related to findings connecting the choice of a social work career with earlier life trauma (Lyter, 2008; Rompf \& Royse, 1994). The current study provides a key followup to research suggesting that trauma histories of helping professionals may affect service provision (Jackson \& Nuttall, 1994; Pearlman \& Mac Ian, 1995; van der Kolk, 1989). In other words, if ACEs increase vulnerability to STS (Ghahramanlou \& Brodbeck, 2000; Nelson-Gardell \& Harris, 2003), VT symptoms (Pearlman \& Mac Ian, 1995), and compassion fatigue (Newell \& MacNeil, 2010), then social service agencies may want to bring attention to prevention of STS, VT, and compassion fatigue, as well as any subsequent negative impact on quality of services, by creating restorative cultures that support staff selfcare. Understanding staff ACE characteristics can help organizations consider ways to prevent further harm to staff while enhancing service quality. Thus, the current study fills an important gap by exploring the full range of ACE categories among workers in child-serving agencies.

ACEs can increase vulnerability to stressors (Larkin \& Records, 2007), and organizational pressures have the potential to become risk factors (Newell \& MacNeil, 2010). In light of current data, agency leaders are encouraged to consider restorative cultures for the workforce that may benefit those served. Operating on the principle of recovery, restorative cultures recognize the important role staff members, both direct and indirect care workers, play in creating a therapeutic community. Staff members rolemodel skills and demonstrate relationship building for clients. To create restorative cultures, leaders engage staff to reflect on practices and goals while orienting to shared values. Staff members are supported in their own selfcare to facilitate the development of restorative cultures (Bloom, 2005b; Larkin, Beckos, \& Shields, 2012).

This approach is bolstered by numerous studies demonstrating that resources can moderate the influence of adversity and foster resilience (Larkin, Felitti, \& Anda, in press). A case study of the Restorative Integral Support (RIS) model demonstrated how one "ACE-informed" organization supported staff self-care as a key element in the restorative culture (Larkin, Beckos, et al., 2012). The Sanctuary Model $^{\circ}$ is an example of an organizational intervention focused on a restorative culture that empha- sizes a collaborative and safe environment (Bloom, 1997, 2005a, 2005b; Rivard, Bloom, McCorkle, \& Abramovitz, 2005). The current findings reinforce the impact that agency culture can have on service providers (Catherall, 1995). Children and families may benefit by increased support to counter ACEs among their providers, including development of restorative organizational cultures.

Anda et al. (2004) also found that ACEs were associated with days of work skipped due to feelings of depression or stress as well as health challenges. Thus, higher ACE Scores within a workforce may have economic implications (Anda et al., 2004). If positive workforce cultures have the potential to counteract vulnerabilities of workers in child-serving agencies with ACEs, this may increase worker performance and reduce health care costs associated with ACEs. The impact of restorative work cultures could be explored through further research. In addition, partnerships with economists may help elucidate cost savings over time (Larkin, Felitti, et al., in press).

\section{Limitations}

The current study is limited by its single agency sample, cross-sectional retrospective design, reliance on selfreport as the only data collection modality, and low response rate $(26 \%)$. Given the exploratory nature of the study, only one residential voluntary child welfare agency in the Northeast was recruited for participation, thereby limiting the generalizability of the findings. Further studies exploring the prevalence of ACEs among other residential voluntary child welfare agencies and other human service agencies would be useful in either validating or disproving the findings from this study.

Although prior studies on the original ACE survey showed strong retrospective reliability of the instrument, an abbreviated ACEs instrument was used for this study, upon which retrospective reliability tests were not conducted. With further use of the abbreviated ACE survey in studies, validity and reliability tests on the instrument would strengthen study findings.

Similarly, Felitti et al. (1998) expressed concern about the hidden nature of ACEs, which may cause underreporting. It is possible that employees with higher ACE Scores chose not to complete the survey, creating a selection bias. Last, the low response rate challenges the validity of the study results.

\section{Implications for Practice, Policy, and Research}

Once ACE prevalence and consequences among the workforce are recognized, several practical steps might be considered to develop and explore restorative cultures. A first step involves raising awareness of ACEs, ACE consequences, resilience, and recovery through educational presentations to staff. Since ethical dilemmas can arise out of unacknowledged VT (Saakvitne \& Pearlman, 
1996), leadership support could help program directors set a tone and example for a positive culture, while policies, procedures, and systems can be developed to facilitate staff self-care within the agency culture.

The workforce includes incoming social work students, suggesting that schools of social work may want to take ACE backgrounds into account. The knowledge and wisdom of social work educators might be usefully applied to the development of restorative cultures within training programs, becoming a subject of future research. Schools of social work can set an example, influencing agency operations and practices.

Future research could explore ACE prevalence among other types of social service providers, as well as assessing STS and VT and examining their relationship to ACEs. Qualitative research could explore provider perspectives on key elements of restorative cultures that reduce STS, VT, and compassion fatigue. This would further inform agencies with restorative cultures seeking to curtail ethical concerns and promote provider effectiveness.

Research could also explore whether ACE backgrounds increase empathy among providers, contribute to an ability to create a compassionate culture, and impact client outcomes. Team-based research partnerships are recommended to determine the effectiveness of ACE-informed programs that include restorative cultures as part of a comprehensive, whole-person approach, including the impact on client outcomes (Larkin, Beckos, \& Martin, in press).

The current study shows a high prevalence of ACEs among a sample of child service providers. Clients may benefit by increased support to workers. Future research recommendations include assessing the impact of restorative cultures on worker well-being, effectiveness, and client outcomes.

\section{References}

Adams, R. E., Boscarino, J. A., \& Figley, C. R. (2006). Compassion fatigue and psychological distress among social workers: A validation study. American Journal of Orthopsychiatry, 76(1), 103-108. doi:10.1037/0002-9432.76.1.103

Anda, R. F., Fleisher, V. I., Felitti, V. J., Edwards, V. J., Whitfield, C. L., Dube, S. R., \& Williamson, D. F. (2004). Childhood abuse, household dysfunction, and indicators of impaired adult worker performance. Permanente Journal, 8(1), 30-38.

Bernstein, D. P., Fink, L., Handelsman, L., Foote, J., Lovejoy, M., Wenzel, K.,...Ruggiero, J. (1994). Initial reliability and validity of a new retrospective measure of child abuse and neglect. American Journal of Psychiatry, 151(8), 1132-1136.

Black, P. N., Jeffreys, D., \& Hartley, E. K. (1993). Personal history of psychosocial trauma in the early life of social work and business students. Journal of Social Work Education, 29(2), 171.

Bloom, S. L. (1997). Creating sanctuary: Toward the evolution of sane societies. New York, NY: Routledge.

Bloom, S. L. (2005a). The Sanctuary Model of organizational change for children's residential treatment. Therapeutic Community, 26(1), 65-81.
Bloom, S. L. (2005b). The Sanctuary Model of trauma-informed organizational change. The Source, 16(1), 12-17. Retrieved from http://www.sanctuaryweb.com/PDFs_new/Bloom\%20 The $\% 20$ Sanctuary $\% 20$ Model $\% 20$ The $\% 20$ Source $\% 20$ Articles\%20Sanctuary.pdf

Bober, T., \& Regehr, C. (2005). Strategies for reducing secondary or vicarious trauma: Do they work? Brief Treatment \& Crisis Intervention, 6(1), 1-9.

Bride, B. E., Radey, M., \& Figley, C. R. (2007). Measuring compassion fatigue. Clinical Social Work Journal, 35(3), 155-163.

Bynum, L., Griffin, T., Ridings, D. L., Wynkoop, K. S., Anda, R. F., Edwards, V. J.,...Croft, J. B. (2010). Adverse childhood experiences reported by adults-five states, 2009. MMWR: Morbidity \& Mortality Weekly Report, 59(49), 1609-1613.

Catherall, D. R. (1995). Preventing institutional secondary traumatic stress disorder. In C. R. Figley (Ed.), Compassion fatigue: Coping with secondary traumatic stress disorder in those who treat the traumatized (pp. 232-247). Levittown, PA: Brunner/ Mazel.

Catherall, D. R. (1999). Coping with secondary traumatic stress: The importance of the therapist's professional peer group. In B. H. Stamm (Ed.), Secondary traumatic stress: Self-care issues for clinicians, researchers, and educators (pp. 80-92). Baltimore, MD: Sidran Press.

Centers for Disease Control and Prevention. (2011). Adverse Childhood Experiences (ACE) Study. Retrieved from http:// www.cdc.gov/ace/index.htm

Chartier, M. J., Walker, J. R., \& Naimark, B. (2010). Separate and cumulative effects of adverse childhood experiences in predicting adult health and health care utilization. Child Abuse \& Neglect, 34(6), 454-464. doi:10.1016/j.chiabu.2009.09.020

Cromer, L. D., \& Freyd, J. J. (2009). Hear no evil, see no evil? Associations of gender, trauma history, and values with believing trauma vignettes. Analyses of Social Issues \& Public Policy, 9(1), 85-96. doi:10.1111/j.1530-2415.2009.01185.x

Dong, M., Anda, R. F., Felitti, V. J., Dube, S. R., Williamson, D. F., Thompson, T. J.,...Giles, W. H. (2004). The interrelatedness of multiple forms of childhood abuse, neglect, and household dysfunction. Child Abuse \& Neglect, 28(7), 771-784. doi:10.1016/j.chiabu.2004.01.008

Dube, S. R., Anda, R. F., Croft, J. B., Edwards, V. J., Giles, W. H., \& Felitti, V. J. (2001). Growing up with parental alcohol abuse: Exposure to childhood abuse, neglect, and household dysfunction. Child Abuse \& Neglect, 25(12), 1627-1640.

Dube, S. R., Williamson, D. F., Thompson, T., Felitti, V. J., \& Anda, R. F. (2004). Assessing the reliability of retrospective reports of adverse childhood experiences among adult HMO members attending a primary care clinic. Child Abuse \& Neglect, 28(7), 729-737. doi:10.1016/j.chiabu.2003.08.009

Dunkley, J., \& Whelan, T. A. (2006). Vicarious traumatisation: Current status and future directions. British Journal of Guidance \& Counselling, 34(1), 107-116. doi:10.1080/03069880500483166

Felitti, V. J., \& Anda, R. F. (2010). The relationship of adverse childhood experiences to adult medical disease, psychiatric disorders and sexual behavior: Implications for healthcare. In R. A. Lanius, E. Vermetten, \& C. Pain (Eds.), The impact of early life trauma on health and disease: The hidden epidemic (pp. 77-87). New York, NY: Cambridge University Press.

Felitti, V. J., Anda, R. F., Nordenberg, D., Williamson, D. F., Spitz, A. M., Edwards, V.,...Marks, J. S. (1998). Relationship of childhood abuse and household dysfunction to many of the leading causes of death in adults. The Adverse Childhood Experiences (ACE) Study. American Journal of Preventive Medicine, 14(4), 245-258.

Figley, C. R. (1995). Compassion fatigue: Coping with secondary traumatic stress disorder in those who treat the traumatized. Levittown, PA: Brunner/Mazel. 
Figley, C. R. (2002). Treating compassion fatigue. New York, NY: Brunner-Routledge.

Follette, V. M., Polusny, M. M., \& Milbeck, K. (1994). Mental health and law enforcement professionals: Trauma history, psychological symptoms, and impact of providing services to child sexual abuse survivors. Professional Psychology: Research and Practice, 25(3), 275-282.

Ga-Young, C. (2011). Organizational impacts on the secondary traumatic stress of social workers assisting family violence or sexual assault survivors. Administration in Social Work, 35(3), 225-242. doi:10.1080/03643107.2011.575333

Ghahramanlou, M., \& Brodbeck, C. (2000). Predictors of secondary trauma in sexual assault trauma counselors. International Journal of Emergency Mental Health, 2(4), 229-240.

Gilbert, R., Widom, C. S., Browne, K., Fergusson, D., Webb, E., \& Janson, S. (2009). Burden and consequences of child maltreatment in high-income countries. Lancet, 373(9657), 68-81.

Green, S. B., \& Salkind, N. J. (2005). Using SPSS for Windows and Macintosh: Analyzing and understanding data (4th ed.). Upper Saddle River, NJ: Pearson Prentice Hall.

Herman, J. L. (1992). Trauma and recovery. New York, NY: Basic Books.

Jackson, H., \& Nuttall, R. (1994). Effects of gender, age, and history of abuse on social workers' judgments of sexual abuse allegations. Social Work Research, 18(2), 105-114.

Knight, C. (2010). Indirect trauma in the field practicum: Secondary traumatic stress, vicarious trauma, and compassion fatigue among social work students and their field instructors. Journal of Baccalaureate Social Work, 15(1), 31-52.

Larkin, H., Beckos, B., \& Martin, E. (in press). Applied integral methodological pluralism: Designing comprehensive social services program evaluation. In S. Esbjorn-Hargens (Ed.), Enacting an integral future. Albany, NY: SUNY.

Larkin, H., Beckos, B., \& Shields, J. (2012). Mobilizing resilience and recovery in response to adverse childhood experiences (ACE): A restorative integral support (RIS) case study. Journal of Prevention \& Intervention in the Community, 40(4), 335-346.

Larkin, H., Felitti, V. J., \& Anda, R. F. (in press). Social work and adverse childhood experiences (ACE) research: Implications for practice and health policy. Social Work in Public Health.

Larkin, H., \& Park, J. (2012). Adverse childhood experiences (ACE), service use, and service helpfulness among people experiencing homelessness. Families in Society: The Journal of Contemporary Social Services, 93(2), 85-93. doi:10.1606/1044-3894.4192

Larkin, H., \& Records, J. (2007). Adverse childhood experiences: Overview, response strategies, and integral theory. Journal of Integral Theory and Practice, 2(3), 1-25.

Lyter, S. C. (2008). Woundedness and social workers: Strategies for supervision of practicum students. Arete, 32(1), 20-37.

McCann, I. L., \& Pearlman, L. A. (1990). Vicarious traumatization: A framework for understanding the psychological effects of working with victims. Journal of Traumatic Stress, 3(1), 131-149.

Munroe, J. F. (1995). Ethical issues associated with secondary trauma in therapists. In B. H. Stamm (Ed.), Secondary traumatic stress: Self-care issues for clinicians, researchers, and educators (pp. 211-229). Lutherville, MD: Sidran Press.

Nelson-Gardell, D., \& Harris, D. (2003). Childhood abuse history, secondary traumatic stress, and child welfare workers. Child Welfare, 82(1), 5-26.

Newell, J. M., \& MacNeil, G. A. (2010). Professional burnout, vicarious trauma, secondary traumatic stress, and compassion fatigue: A review of theoretical terms, risk factors, and preventive methods for clinicians and researchers. Best Practice in Mental Health, 6(2), 57-68.
Nunnally, J. (1978). Psychometric theory. New York, NY: McGrawHill.

Pearlman, L. A., \& Mac Ian, P. S. (1995). Vicarious traumatization: An empirical study of the effects of trauma work on trauma therapists. Professional Psychology: Research and Practice, 26(6), 558-565.

Rivard, J. C., Bloom, S. L., McCorkle, D., \& Abramovitz, R. (2005). Preliminary results of a study examining the implementation and effects of a trauma recovery framework for youths in residential treatment. Therapeutic Community, 26(1), 83-96.

Rompf, E. L., \& Royse, D. (1994). Choice of social work as a career: Possible influences. Journal of Social Work Education, 30(2), 163-171.

Rothschild, B., \& Rand, M. (2006). Help for the helper, self-care strategies for managing burnout and stress: The psychophysiology of compassion fatigue and vicarious trauma. New York, NY: W.W. Norton.

Russel, R., Gill, P., Coyne, A., \& Woody, J. (1993). Dysfunction in the family of origin of MSW and other graduate students. Journal of Social Work Education, 29(1), 121-129.

Saakvitne, K. W., \& Pearlman, L. A. (1996). Transforming the pain: A workbook on vicarious traumatization. New York, NY: Norton.

Sabin-Farrell, R., \& Turpin, G. (2003). Vicarious traumatization: Implications for the mental health of health workers? Clinical Psychology Review, 23(3), 449. doi:10.1016/s02727358(03)00030-8

Schauben, L. J., \& Frazier, P. A. (1995). Vicarious trauma: The effects on female counselors of working with sexual violence survivors. Psychology of Women Quarterly, 19(1), 49-64. doi:10.1111/j.1471-6402.1995.tb00278.x

Straus, M., \& Gelles, R. J. (1990). Physical violence in American families: Risk factors and adaptations to violence in 8,145 families. New Brunswick, NJ: Transaction Press.

Trippany, R. L., White Kress, V. E., \& Wilcoxen, S. A. (2004). Preventing vicarious trauma: What counselors should know when working with trauma survivors. Journal of Counseling \& Development, 82(Winter), 31-37.

van der Kolk, B. A. (1989). The compulsion to repeat the trauma. Re-enactment, revictimization, and masochism. Psychiatric Clinics of North America, 12(2), 389-411.

Wyatt, G. E. (1985). The sexual abuse of Afro-American and WhiteAmerican women in childhood. Child Abuse \& Neglect, 9(4), 507-519.

Nina Esaki, PhD, MBA, MSW, director of research, Sanctuary Institute, Andrus. Heather Larkin, PhD, LCSW-R, assistant professor, University at Albany. Correspondence: NEsaki@jdam.org; Andrus, 1156 North Broadway, Yonkers, NY 10701.

Authors' note. We extend our gratitude to the child welfare agency staff whose provision of valuable information about their personal histories allowed us to conduct this study.

Manuscript received: October 25, 2011

Revised: March 28, 2012

Accepted: May 1, 2012

Disposition editor: Christopher G. Petr 